\title{
Plurisubharmonic functions on hypercomplex manifolds and HKT-geometry.
}

\author{
Semyon Alesker, Misha Verbitsky ${ }^{\dagger}$
}

\begin{abstract}
A hypercomplex manifold is a manifold equipped with a triple of complex structures $I, J, K$ satisfying the quaternionic relations. We define a quaternionic analogue of plurisubharmonic functions on hypercomplex manifolds, and interpret these functions geometrically as potentials of HKT (hyperkähler with torsion) metrics, and prove a quaternionic analogue of A.D. Aleksandrov and Chern-Levine-Nirenberg theorems.
\end{abstract}

\section{Contents}

1 Introduction. 2

$\begin{array}{llr}2 & \text { Some linear algebra. } & 6\end{array}$

3 Differential operators on hypercomplexmanifolds. 15

4 Comparison with the flat case. 16

5 Vector bundles with a cone. 20

6 Quaternionic plurisubharmonic functionson $\mathbb{H}^{n}$. 21

7 Plurisubharmonic functions on hypercomplex manifolds. 23

*Partially supported by ISF grant $1369 / 04$.

${ }^{\dagger}$ Misha Verbitsky is an EPSRC advanced fellow supported by CRDF grant RM1-2354MO02 and EPSRC grant GR/R77773/01 


\section{Introduction.}

The goal of this article is to introduce a class of (continuous) quaternionic plurisubharmonic functions on hypercomplex manifolds. We prove a version of A.D. Aleksandrov and Chern-Levine-Nirenberg theorems for it. Then we present a geometric characterization of smooth quaternionic strictly plurisubharmonic functions as (local) potentials of so called HKT-metrics on hypercomplex manifolds (HKT is the abbreviation of HyperKähler with Torsion). This interpretation is analogous to the well known interpretation of smooth complex strictly plurisubharmonic functions on complex manifolds as (local) potentials of Kähler metrics.

The class of quaternionic plurisubharmonic functions on the flat space $\mathbb{H}^{n}$ was introduced by one of the authors in [A1] and independently by G. Henkin [He] (unpublished). This class was studied in [A1, [A2, A3]. Applications to the theory of valuations on convex sets were obtained in A3. In this article we extend some of those definitions and results to hypercomplex manifolds.

Other results related to quaternionic pluripotential theory on hypercomplex manifolds were obtained by one of the authors [V1 (e.g. a quaternionic version of Sibony's lemma Sib] on extensions of positive currents and a version of the Skoda-El Mir theorem).

Let us discuss the main results of this article in greater detail.

1.1 Definition. A hypercomplex manifold is a smooth manifold $X$ together with a triple $(I, J, K)$ of complex structures satisfying the usual quaternionic relations:

$$
I J=-J I=K \text {. }
$$

1.2 Remark. (1) We will suppose in this article (in the opposite to much of the literature on the subject) that the complex structures $I, J, K$ act on the right on the tangent bundle $T X$ of $X$. This action extends uniquely to the right action of the algebra $\mathbb{H}$ of quaternions on $T X$.

(2) It follows that the dimension of a hypercomplex manifold $X$ is divisible by 4 .

(3) Hypercomplex manifolds were introduced explicitly by Boyer [Bo]

Let $\left(X^{4 n}, I, J, K\right)$ be a hypercomplex manifold. Let us denote by $\Lambda_{I}^{p, q}(X)$ the vector bundle of $(p, q)$-forms on the complex manifold $(X, I)$. By the 
abuse of notation we will also denote by the same symbol $\Lambda_{I}^{p, q}(X)$ the space of $C^{\infty}$-sections of this bundle.

Let

$$
\partial: \Lambda_{I}^{p, q}(X) \rightarrow \Lambda_{I}^{p+1, q}(X)
$$

be the usual $\partial$-differential of differential forms on the complex manifold $(X, I)$.

Set

$$
\partial_{J}:=J^{-1} \circ \bar{\partial} \circ J
$$

1.3 Claim ([V2]). (1) $J: \Lambda_{I}^{p, q}(X) \rightarrow \Lambda_{I}^{q, p}(X)$.

(2) $\partial_{J}: \Lambda_{I}^{p, q}(X) \rightarrow \Lambda_{I}^{p+1, q}(X)$.

(3) $\partial \partial_{J}=-\partial_{J} \partial$

1.4 Remark. Claim 1.3 (1) is clear because $I$ and $J$ anticommute, and Claim 1.3 (2) is directly implied by Claim $1.3(1)$.

1.5 Definition ([V2] $)$. Let $k=0,1, \ldots, n$. A form $\omega \in \Lambda_{I}^{2 k, 0}(X)$ is called real if

$$
\overline{J \circ \omega}=\omega
$$

We will denote the subspace of real $C^{\infty}$-smooth $(2 k, 0)$-forms on $(X, I)$ by $\Lambda_{I, \mathbb{R}}^{2 k, 0}(X)$.

1.6 Lemma. Let $X$ be a hypercomplex manifold. Let $f: X \rightarrow \mathbb{R}$ be a smooth function. Then $\partial \partial_{J} f \in \Lambda_{I, \mathbb{R}}^{2,0}(X)$.

This lemma is proved in Section 3 as Lemma 3.3

1.7 Definition. Let $\omega \in \Lambda_{I, \mathbb{R}}^{2,0}(X)$. Let us say that $\omega$ is non-negative (notation: $\omega \geq 0)$ if

$$
\omega(Y, Y \circ J) \geq 0
$$

for any (real) vector field $Y$ on the manifold $X$. Equivalently, $\omega$ is nonnegative if $\omega(Z, \bar{Z} \circ J) \geq 0$ for any $(1,0)$-vector field $Z$.

1.8 Definition. A continuous function

$$
h: X \rightarrow \mathbb{R}
$$

is called quaternionic plurisubharmonic if $\partial \partial_{J} h$ is a non-negative (generalized) section of $\Lambda_{I, \mathbb{R}}^{2,0}(X)$. 
1.9 Remark. The non-negativity in the generalized sense is discussed in detail in Section 5 .

Let us denote by $P^{\prime}(X)$ the class of continuous quaternionic plurisubharmonic functions on $X$. Let us denote by $P^{\prime \prime}(X)$ the subclass of functions from $P^{\prime}(X)$ with the following additional property: a function $h \in P^{\prime}(X)$ belongs to $P^{\prime \prime}(X)$ if and only if any $x \in X$ has a neighborhood $U \ni x$ and a sequence $\left\{h_{N}\right\} \subset P^{\prime}(U) \cap C^{2}(U)$ such that $h_{N} \stackrel{C^{0}(U)}{\rightarrow} h$ (where the convergence is understood in sense of the uniform convergence on compact subsets of $U$ ). Thus $P^{\prime \prime}(X) \subset P^{\prime}(X)$.

We conjecture that $P^{\prime}(X)=P^{\prime \prime}(X)$. This conjecture is true when $X$ is an open subset of $\mathbb{H}^{n}$.

The first main result of the article is the following theorem.

1.10 Theorem. Let $X$ be a hypercomplex manifold of (real) dimension $4 n$. Let $0<k \leq n$. For any $h_{1}, \ldots, h_{k} \in P^{\prime \prime}(X)$ one can define a non-negative generalized section of $\Lambda_{I, \mathbb{R}}^{2 k}$ denoted by $\partial \partial_{J} h_{1} \wedge \cdots \wedge \partial \partial_{J} h_{k}$ which is uniquely characterized by the following two properties:

(1) if $h_{1}, \ldots, h_{k} \in C^{2}(X)$ then the definition is clear;

(2) if $\left\{h_{i}^{N}\right\} \subset P^{\prime \prime}(X), h_{i}^{N} \stackrel{C^{0}}{\rightarrow} h_{i}$ as $N \rightarrow \infty, i=1, \ldots, k$, then $h_{i} \in P^{\prime \prime}(X)$ and

$$
\partial \partial_{J} h_{1}^{N} \wedge \cdots \wedge \partial \partial_{J} h_{k}^{N} \rightarrow \partial \partial_{J} h_{1} \wedge \cdots \wedge \partial \partial_{J} h_{k}
$$

in the weak topology on measures.

This theorem is proved in Section 7 as Theorem [7.9]

1.11 Remark. Theorem 1.10 is a quaternionic analogue of a (real) result of A.D. Aleksandrov Al and a (complex) result of Chern-Levine-Nirenberg CLL. When the hypercomplex manifold $X$ is an open subset of the flat space $\mathbb{H}^{n}$ Theorem [1.10 was proved by one of the authors in [A3], and in a special case of $k=n$ in [A1]. Some applications of this theorem in the flat case to the theory of valuations on convex sets were obtained in [A3].

In order to formulate the second main result we have to remind the definition of an HKT-metric on a hypercomplex manifold $X$. Let $g$ be a Riemannian metric on $X$. The metric $g$ is called quaternionic Hermitian (or hyperhermitian) if $g$ is invariant with respect to the group $S U(2) \subset \mathbb{H}^{*}$ of unitary quaternions. 
Given a quaternionic Hermitian metric $g$ on a hypercomplex manifold $X$, consider the differential form

$$
\Omega:=\omega_{J}-\sqrt{-1} \omega_{K}
$$

where $\omega_{L}(A, B):=g(A, B \circ L)$ for any $L \in \mathbb{H}$ with $L^{2}=-1$ and any vector fields $A, B$ on $X$. It is easy to see that $\Omega$ is a $(2,0)$-form with respect to the complex structure $I$.

1.12 Definition. The metric $g$ on $X$ is called HKT-metric if

$$
\partial \Omega=0
$$

1.13 Remark. HKT-metric on hypercomplex manifolds first were introduced by Howe and Papadopoulos $[\mathrm{HP}$. Their original definition was different but equivalent to Definition 1.12 (see [GP]).

Let us denote by $S_{\mathbb{H}}(X)$ the vector bundle over $X$ such that its fiber over a point $x \in X$ is equal to the space of hyperhermitian forms on the tangent space $T_{x} X$ (see Definition 2.2 in Section 2). Consider the map of vector bundles

$$
t: \Lambda_{I, \mathbb{R}}^{2,0}(X) \rightarrow S_{\mathbb{H}}(X)
$$

defined by $t(\eta)(A, A)=\eta(A, A \circ J)$ for any vector field $A$ on $X$. The $t$ is an isomorphism of vector bundles (this was proved in V2] see also Lemma 2.10 below).

The second main result is the following observation which provides a geometric interpretation of the notion of quaternionic (strictly) plurisubharmonic function on a hypercomplex manifold.

1.14 Proposition. (1) Let $f$ be an infinitely smooth strictly plurisubharmonic function on a hypercomplex manifold $(X, I, J, K)$. Then $t\left(\partial \partial_{J} f\right)$ is an HKT-metric.

(2) Conversely assume that $g$ is an HKT-metric. Then any point $x \in$ $X$ has a neighborhood $U$ and an infinitely smooth strictly plurisubharmonic function $f$ on $U$ such that $g=t\left(\partial \partial_{J} f\right)$ in $U$.

This result is a direct consequence of a quaternionic version of the local $\partial \bar{\partial}$-lemma well known for the complex manifolds. We call this generalization local $\partial \partial_{J}$-lemma. It says as follows. 
1.15 Proposition. Let $\Omega \in C^{\infty}\left(X, \Lambda_{I, \mathbb{R}}^{2,0}\right)$. Then locally on $X$ the form $\Omega$ can be presented in a form

$$
\Omega=\partial \partial_{J} f
$$

with $f$ being a $C^{\infty}$-smooth real valued function if and only if $\partial \Omega=0$.

The proof of this result is a rather straightforward application of the main theorem of [BS] (which uses in turn [MCSa]).

The article is organized as follows. In Section 2 we discuss some auxiliary constructions from quaternionic linear algebra.

In Section 3 we discuss differential operators $\partial$ and $\partial_{J}$ on differential forms on general hypercomplex maifolds and the so called Dirac operators on $\mathbb{H}^{n}$.

In Section 4 we make a comparison between differential operators on the flat space $\mathbb{H}^{n}$ and on general hypercomplex manifolds; the goal is to rewrite some expressions on $\mathbb{H}^{n}$ in a language working in the more general setting of hypercomplex manifolds.

In Section 5 we introduce a general notion of a vector bundle with a cone in order to have a notion of positive (with respect to this cone) section of the vector bundle.

In Section [6 we remind the definition and some results on quaternionic plurisubharmonic functions on the flat space $\mathbb{H}^{n}$ following A1, A3.

Section 7 contains the main definitions of this article and the proof of the first main result Theorem 1.10,

Section 8 describes the relation between quaternionic plurisubharmonic functions and HKT-geometry. Namely we prove Proposition 1.14 and the local $\partial \partial_{J}$-lemma (Proposition 8.1).

Acknowledgements. The first named author is grateful to G. Henkin and V. Shevchishin for useful discussions. We thank the referee for important remarks on the first version of the article.

\section{Some linear algebra.}

In this section we describe some facts from linear algebra.

2.1 Definition. (1) Let $A=\left(a_{i j}\right)$ be an $(n \times n)$-matrix with quaternionic entries. Then $A$ is called hyperhermitian if

$$
a_{i j}=\bar{a}_{j i}
$$


where $\bar{q}$ is the usual conjugation of a quaternion $q$.

(2) A hyperhermitian matrix $A$ is called non-negative definite (resp. positive definite) if for any $\xi \in \mathbb{H}^{n} \backslash\{0\}$ one has $\xi^{*} A \xi \geq 0$ (resp. $\left.\xi^{*} A \xi>0\right)$.

Let $V$ be a right vector space over quaternions.

2.2 Definition. A hyperhermitian semilinear form on $V$ is a map $a: V \times$ $V \rightarrow \mathbb{H}$ satisfying the following properties:

(a) $a$ is additive with respect to each argument;

(b) $a(x, y \cdot q)=a(x, y) \cdot q$ for any $x, y \in V$ and any $q \in \mathbb{H}$;

(c) $a(x, y)=\overline{a(y, x)}$.

2.3 Remark. It is easy to see that any hyperhermitian form $a$ on $\mathbb{H}^{n}$ can be written in the form $a(X, Y)=\sum_{i, j=1}^{n} \bar{x}_{i} a_{i j} y_{j}$ where $\left(a_{i j}\right)$ is a uniquely determined hyperhermitian $n \times n$ - matrix.

The space of hyperhermitian forms on $V$ we will denote by $S_{\mathbb{H}}(V)$.

For a quaternionic $n \times n$-matrix $A \in M_{n}(\mathbb{H})$ let us denote by ${ }^{\mathbf{R}} A$ the realization matrix of $A$ which is a real $4 n \times 4 n$-matrix. (To construct it, consider $A$ as a matrix of a quaternionic transformation $\mathbb{H}^{n} \rightarrow \mathbb{H}^{n}$. Identify $\mathbb{H}^{n} \sim \mathbb{R}^{4 n}$ in the standard way. Then $\mathbf{R}_{A}$ is the matrix of this transformation with respect to the standard basis of $\mathbb{R}^{4 n}$.)

The following result is classical (see $\mathrm{As}$ for the references).

2.4 Theorem. There exists a polynomial $P$ defined on the space of all hyperhermitian $n \times n$-matrices such that for any hyperhermitian $n \times n$-matrix $A$ one has $\operatorname{det}\left({ }^{\boldsymbol{R}} A\right)=P^{4}(A)$ and $P(I d)=1 . P$ is defined uniquely by these two properties. Furthermore $P$ is homogeneous of degree $n$ and has integer coefficients.

Thus for any hyperhermitian matrix $A$ the value $P(A)$ is a real number, and it is called the Moore determinant of the matrix $A$. The explicit formula for the Moore determinant was given by Moore [M] (see also the survey $\mathrm{As}$. and [GRW]). From now on the Moore determinant of a matrix $A$ will be denoted by $\operatorname{det} A$. This notation should not cause any confusion with the usual determinant of real or complex matrices due to part (i) of the next theorem.

2.5 Theorem. (i) The Moore determinant of any complex hermitian matrix considered as quaternionic hyperhermitian matrix is equal to its usual determinant. 
(ii) For any hyperhermitian matrix $A$ and any quaternionic matrix $C$

$$
\operatorname{det}\left(C^{*} A C\right)=\operatorname{det} A \cdot \operatorname{det}\left(C^{*} C\right) .
$$

For the proof we refer to A1 though this result was known earlier and is probably a folklore.

2.6 Example. (a) Let $A=\operatorname{diag}\left(\lambda_{1}, \ldots, \lambda_{n}\right)$ be a diagonal matrix with real $\lambda_{i}$ 's. Then $A$ is hyperhermitian and the Moore $\operatorname{determinant} \operatorname{det} A=\prod_{i} \lambda_{i}$.

(b) A general hyperhermitian $2 \times 2$ matrix $A$ has the form

$$
A=\left[\begin{array}{cc}
a & q \\
\bar{q} & b
\end{array}\right]
$$

where $a, b \in \mathbb{R}, q \in \mathbb{H}$. Then $\operatorname{det} A=a b-q \bar{q}(=a b-\bar{q} q)$.

Let $V$ be a right $\mathbb{H}$-module of quaternionic dimension $n$. Let $0 \leq k \leq n$ be an integer. Let us denote by

$$
\Lambda_{I}^{2 k, 0}(V):=\wedge_{\mathbb{C}}^{2 k}\left(V_{I}^{*}\right)
$$

where $V_{I}$ is the space $V$ equipped with the complex structure $I, V_{I}^{*}$ is its dual. Note that

$$
J: V_{I} \rightarrow V_{I}
$$

is an anti-linear map (namely $J(x \cdot \lambda)=J(x) \cdot \bar{\lambda})$. It induces an anti-linear involution

$$
J: \Lambda_{I}^{2 k, 0}(V) \rightarrow \Lambda_{I}^{2 k, 0}(V) .
$$

Let us denote by $\Lambda_{I, \mathbb{R}}^{2 k, 0}(V)$ the real subspace fixed by this involution.

2.7 Lemma. The natural representation of the group $G L_{n}(\mathbb{H})$ in $\Lambda_{I, \mathbb{R}}^{2 k, 0}(V)$ is absolutely irreducible (in particular it is irreducible). The complexification of this representation has highest weight $(\underbrace{0, \ldots, 0}_{2(n-k) \text { times }}, \underbrace{-1, \ldots,-1}_{2 k \text { times }})$ as a representation of $G L_{n}(\mathbb{H}) \otimes_{\mathbb{R}} \mathbb{C}=G L_{2 n}(\mathbb{C})$.

To prove Lemma 2.7 we will need the following elementary lemma which is in fact a special case of Hilbert 90 Theorem. 
2.8 Lemma. Let $W$ be a complex vector space. Let $\sigma: W \rightarrow W$ be an anti-linear involution of $W$. Then

$$
W=W^{\sigma} \oplus W^{\sigma} \cdot \sqrt{-1}
$$

where $W^{\sigma}$ is the (real) subspace of $\sigma$-fixed vectors.

Proof of Lemma 2.7 assuming Lemma 2.8. By Lemma 2.8] we have

$$
\Lambda_{I}^{2 k, 0}(V)=\Lambda_{I, \mathbb{R}}^{2 k, 0}(V) \oplus \Lambda_{I, \mathbb{R}}^{2 k, 0}(V) \cdot I .
$$

Hence $\Lambda_{I}^{2 k, 0}(V)$ is the complexification of the representation of $G L_{n}(\mathbb{H})$ in $\Lambda_{I, \mathbb{R}}^{2 k, 0}(V)$. The complexification of the group $G L_{n}(\mathbb{H})$ is the group $G L_{2 n}(\mathbb{C})$. But the representation of the group $G L_{2 n}(\mathbb{C})$ in $\Lambda_{I}^{2 k, 0}(V)=\wedge_{\mathbb{C}}^{2 k}\left(V_{I}^{*}\right)$ is irreducible with highest weight $(\underbrace{0, \ldots, 0}, \underbrace{-1, \ldots,-1})$ (this is a basic fact from representation theory). Q.E.D.

$$
2(n-k) \text { times } 2 k \text { times }
$$

Proof of Lemma 2.7. Set

$$
W^{\sigma^{\prime}}:=\{x \in W \mid \sigma x=-x\} .
$$

Then clearly $W=W^{\sigma} \oplus W^{\sigma^{\prime}}$. Obviously $x \in W^{\sigma}$ if and only if $x \cdot I \in W^{\sigma^{\prime}}$, hence $W^{\sigma^{\prime}}=W^{\sigma} \cdot I$. Q.E.D.

2.9 Lemma. Let $\omega \in \Lambda_{I, \mathbb{R}}^{2,0}(V)$. Define a quadratic form

$$
B(X, X)=\omega(X, X \circ J) .
$$

Then $B$ is hyperhermitian, i.e. $B \in S_{\mathbb{H}}(V)$.

Proof. First let us check that $B$ is real valued. Indeed

$$
\begin{array}{r}
\overline{B(X, X)}=\overline{\omega(X, X \circ J)}=\omega\left(X \circ J, X \circ J^{2}\right)= \\
\omega(X, X \circ J)=B(X, X) .
\end{array}
$$

Let us check that $B$ is invariant under $I, J, K$ :

$$
\begin{array}{r}
B(X \circ I, X \circ I)=\omega(X \circ I, X \circ I J)= \\
-\omega(X \circ I,(X \circ J) \circ I)=\omega(X, X \circ J)=B(X, X) ; \\
B(X \circ J, X \circ J)=\omega(X \circ J,-X)=\omega(X, X \circ J)=B(X, X) ; \\
B(X \circ K, X \circ K)=B(X \circ I J, X \circ I J)=B(X \circ I, X \circ I)=B(X, X) .
\end{array}
$$


Q.E.D.

Thus Lemma 2.9] defines a map

$$
t: \Lambda_{I, \mathbb{R}}^{2,0}(V) \rightarrow S_{\mathbb{H}}(V)
$$

2.10 Lemma ([V2]). The map $t$ defined in (2.1) is an isomorphism. The inverse map is given by

$$
\left(t^{-1} g\right)(X, Y)=-(g(X, Y \circ J)-\sqrt{-1} g(X, Y \circ K)) .
$$

Proof. Let us consider the map

$$
\phi: S_{\mathbb{H}}(V) \rightarrow \Lambda_{I, \mathbb{R}}^{2,0}(V)
$$

defined by $(\phi(g))(X, Y)=-(g(X, Y \circ J)-\sqrt{-1} g(X, Y \circ K))$. We will show that $\phi$ is the inverse of $t$. But first let us check that indeed for any $g \in S_{\mathbb{H}}(V)$ we have

$$
\phi(g) \in \Lambda_{I, \mathbb{R}}^{2,0}(V)
$$

To prove that $\phi(g) \in \Lambda_{I}^{2,0}(V)$ it is enough to check that

$$
\left.\frac{d}{d \theta}\right|_{0}(\phi(g))\left(X \circ e^{\theta I}, Y \circ e^{\theta I}\right)=2 \sqrt{-1}(\phi(g))(X, Y) .
$$

We have

$$
\begin{aligned}
&\left.\frac{d}{d \theta}\right|_{0}(\phi(g))\left(X \circ e^{\theta I}, Y \circ e^{\theta I}\right)=\phi(g)(X \circ I, Y)+\phi(g)(X, Y \circ I) \\
&=-(g(X \circ I, Y \circ J)-\sqrt{-1} g(X \circ I, Y \circ K)+g(X, Y \circ I J)-\sqrt{-1} g(X, Y \circ I K)) \\
&=-(g(X, Y \circ K)+\sqrt{-1} g(X, Y \circ J)+g(X, Y \circ K)+\sqrt{-1} g(X, Y \circ J)) \\
&=2 \sqrt{-1}(\phi(g))(X, Y)
\end{aligned}
$$

Let us show that $\phi(g)$ is real. We have

$$
\begin{aligned}
& \overline{(\phi(g))(X \circ J, Y \circ J)} \\
& \quad=-\left(g\left(X \circ J, Y \circ J^{2}\right)+\sqrt{-1} g(X \circ J, Y \circ J K)\right)=(\phi(g))(X, Y) .
\end{aligned}
$$

Thus (2.3) is proved. 
Let $g \in S_{\mathbb{H}}(V)$. Then

$$
(t \circ \phi)(g)(X, X)=-\left(g\left(X, X \circ J^{2}\right)-\sqrt{-1} g(X, X \circ J K)\right)=g(X, X) .
$$

Hence

$$
t \circ \phi=i d
$$

Let us check that $\phi \circ t=i d$. Let $\eta \in \Lambda_{I, \mathbb{R}}^{2,0}(V)$. Set $g:=t(\eta)$. Then

$$
\begin{aligned}
& g(X, Y)=\frac{1}{2}(g(X+Y, X+Y)-g(X, X)-g(Y, Y))= \\
& \frac{1}{2}(\eta(X+Y,(X+Y) \circ J)-\eta(X, X \circ J)-\eta(Y, Y \circ J)) .
\end{aligned}
$$

Then

$$
\begin{aligned}
(\phi \circ t)(\eta)(X, Y)=-(g(X, Y \circ J)-\sqrt{-1} g(X, Y \circ K)) & \\
= & -\frac{1}{2}(g(X+Y \circ J, X+Y \circ J)-g(X, X)-g(Y, Y)- \\
& \sqrt{-1}(g(X+Y \circ K, X+Y \circ K)-g(X, X)-g(Y, Y))) \\
= & -\frac{1}{2}(\eta(X+Y \circ J, X \circ J-Y)-\eta(X, X \circ J)-\eta(Y, Y \circ J)- \\
& \sqrt{-1}(\eta(X+Y \circ K, X \circ J-Y \circ I)-\eta(X, X \circ J)-\eta(Y, Y \circ J))) .
\end{aligned}
$$

Opening by bilinearity and making cancellations the last expression becomes

$$
\begin{aligned}
& -\frac{1}{2}(-\eta(X, Y)-\eta(X \circ J, Y \circ J)- \\
& \sqrt{-1}(-\eta(X, Y \circ I)+\eta(Y \circ K, X \circ J)-\eta(Y \circ I J, Y \circ I)+\eta(Y \circ K, Y \circ I))) \\
& \quad=\frac{1}{2}(\eta(X, Y)+\overline{\eta(X, Y)}-\sqrt{-1}(\eta(X, Y \circ I)-\overline{\eta(Y \circ I, X)}) \\
& \quad=\operatorname{Re} \eta(X, Y)-\sqrt{-1} \operatorname{Re} \eta(X, Y \circ I)=\eta(X, Y) .
\end{aligned}
$$

Thus Lemma 2.10 is proved. Q.E.D.

Now we are going to define convex cones of strongly and weakly positive elements in $\Lambda_{I, \mathbb{R}}^{2 k, 0}(V), 0 \leq k \leq n$. The exposition is analogous to the complex case as in Harvey [Ha] (see also Lelong $\left[\mathrm{L}\right.$ ). First observe that $\Lambda_{I, \mathbb{R}}^{2 n, 0}(V)$ is a real one-dimensional real space. It is canonically oriented. Let us denote the (closed) half line of positive elements in $\Lambda_{I, \mathbb{R}}^{2 n, 0}(V)$ by $\Lambda_{I, \mathbb{R}}^{2 n, 0}(V)_{\geq 0}$. 
2.11 Definition. (1) An element $\eta \in \Lambda_{I, \mathbb{R}}^{2 k, 0}(V)$ is called strongly positive if it can be presented as a finite sum of elements of the form $f^{*} \xi$ where $f: V \rightarrow U$ is a morphism of right $\mathbb{H}$-modules, $\operatorname{dim}_{\mathbb{H}} U=k, \xi \in \Lambda_{I, \mathbb{R}}^{2 k, 0}(U)_{\geq 0}$.

(2) An element $\eta \in \Lambda_{I, \mathbb{R}}^{2 k, 0}(V)$ is called weakly positive if for any strongly positive element $\zeta \in \Lambda_{I, \mathbb{R}}^{2(n-k), 0}(V)$ one has $\eta \wedge \zeta \in \Lambda_{I, \mathbb{R}}^{2 n, 0}(V)_{\geq 0}$.

It is clear that strongly and weakly positive elements form convex cones. Let us denote by $C^{k}(V)$ (resp. $K^{k}(V)$ ) the cone of strongly (resp. weakly) positive elements.

2.12 Remark. (1) Clearly we have $\left(\Lambda_{I, \mathbb{R}}^{2 k, 0}(V)\right)^{*}=\Lambda_{I, \mathbb{R}}^{2(n-k), 0}(V) \otimes \Lambda_{I, \mathbb{R}}^{2 n, 0}$. Then the closure $\overline{C^{k}(V)}$ is the cone dual to the cone $K^{n-k} \otimes \Lambda_{I, \mathbb{R}}^{2 n, 0}(V)_{\geq 0}$. (The duality of cones is understood in the standard sense: for a convex cone $K$ in a vector space $W$ one defines the dual cone $K^{\circ}:=\left\{y \in W^{*} \mid y(x) \geq 0 \forall x \in\right.$ $K\}$.

(2)We will see below in Propositions 2.13)(2), 2.18 that $\overline{C^{2}(V)}=K^{2}(V)$ and this cone coincides with the cone $\left\{\eta \in \Lambda_{I, \mathbb{R}}^{2,0}(V) \mid \eta(A, A \circ J) \geq 0 \forall A \in V\right\}$.

Let us state some basic properties of the cones $C^{k}(V), K^{k}(V)$.

2.13 Proposition. (1) $C^{k}(V) \subset K^{k}(V)$.

(2) $C^{k}(V) \wedge C^{l}(V) \subset C^{k+l}(V)$.

(3) For $k=0,1, n-1, n$

$$
C^{k}(V)=K^{k}(V)
$$

(4) The cones $C^{k}(V)$ and $K^{k}(V)$ have non-empty interior.

This proposition was proved in [A3] in a somewhat different language. Now we will do this comparison of languages.

First let us describe the relevant linear algebraic constructions from [A3], Section 2. Remind that we denote by $S_{\mathbb{H}}(V)$ the space of hyperhermitian forms on $V$. Recall $\operatorname{dim}_{\mathbb{H}} V=n$.

2.14 Proposition. [[A3], Section 2] Let $0 \leq k \leq n$ be an integer. The space $\operatorname{Sym}_{\mathbb{R}}^{k}\left(S_{\mathbb{H}}(V)\right)$ has a unique quotient denoted by $\Omega^{k, k}(V)$ such that the complexification of the natural representation of $G L_{n}(\mathbb{H})$ in $\Omega^{k, k}(V)$ is irreducible and has highest weight $(\underbrace{0, \ldots, 0}_{2(n-k) \text { times }}, \underbrace{-1, \ldots,-1}_{2 k \text { times }})$ as a representation of $G L_{2 n}(\mathbb{C})=G L_{n}(\mathbb{H}) \otimes_{\mathbb{R}} \mathbb{C}$. 
Let

$$
p_{k}: \operatorname{Sym}^{k}\left(S_{\mathbb{H}}(V)\right) \rightarrow \Omega^{k, k}(V)
$$

be the canonical projection.

Define

$$
\Omega^{\bullet}(V):=\oplus_{k=0}^{n} \Omega^{k, k}(V) .
$$

Let us describe the algebra structure on $\Omega^{\bullet}(V)$ following [A3]. Consider the composition $\mu$ of maps

$$
\operatorname{Sym}^{k}\left(S_{\mathbb{H}}(V) \otimes \operatorname{Sym}^{l}\left(S_{\mathbb{H}}(V)\right) \rightarrow \operatorname{Sym}^{k+l}\left(S_{\mathbb{H}}(V)\right) \stackrel{p_{k+l}}{\rightarrow} \Omega^{k+l, k+l}(V) .\right.
$$

It was shown in Proposition 2.1.11 of A3 that $\mu$ factorizes (uniquely) via $p_{k} \otimes p_{l}$, namely there exists a unique map

$$
m: \Omega^{k, k}(V) \otimes \Omega^{l, l}(V) \rightarrow \Omega^{k+l, k+l}(V)
$$

which makes the following diagram commutative:

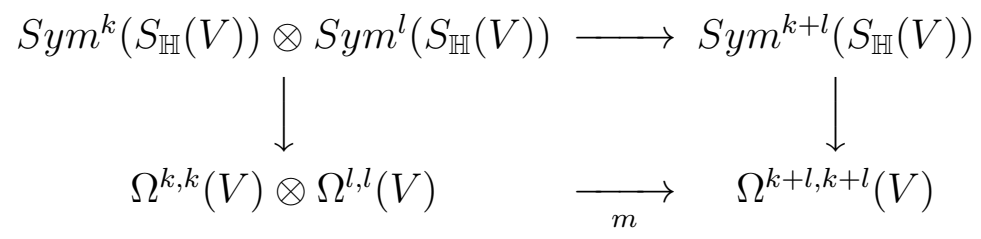

This map $m$ defines the product on $\Omega^{\bullet}(V)$.

2.15 Proposition ([A3], Theorem 2.1.13). The correspondence

$$
V \mapsto \Omega^{\bullet}(V)
$$

is a contravariant functor from the category of finite dimensional $\mathbb{H}$-modules to the category of finite dimensional commutative associative graded algebras. For a fixed $V$ the graded algebra $\Omega^{\bullet}(V)$ satisfies the Poincaré duality.

Recall that we have the isomorphism

$$
t: \Lambda_{I, \mathbb{R}}^{2,0} \stackrel{\sim}{\rightarrow} S_{\mathbb{H}}(V) .
$$

Clearly $t$ commutes with the natural action of $G L_{n}(\mathbb{H})$. Fix an integer $0 \leq$ $k \leq n$. We have the canonical map

$$
\gamma_{k}: \operatorname{Sym}^{k}\left(\Lambda_{I, \mathbb{R}}^{2,0}(V)\right) \rightarrow \Lambda_{I, \mathbb{R}}^{2 k, 0}(V)
$$

given by $\eta_{1} \otimes \cdots \otimes \eta_{k} \mapsto \eta_{1} \wedge \cdots \wedge \eta_{k}$. 
2.16 Lemma. There exists a unique map

$$
\tau_{k}: \Lambda_{I, \mathbb{R}}^{2 k, 0}(V) \rightarrow \Omega^{k, k}(V)
$$

which makes the following diagram commutative:

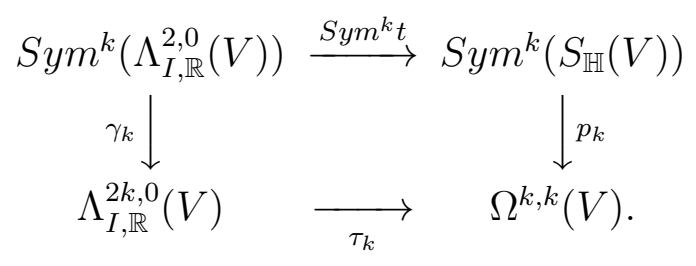

the map $\tau_{k}$ is an isomorphism and commutes with the action of $G L_{n}(\mathbb{H})$.

Proof. This lemma follows immediately from the following fact:

(a) $t$ is isomorphism of $G L_{n}(\mathbb{H})$-modules;

(b) $\operatorname{Sym}^{k}\left(S_{\mathbb{H}}(V)\right) \otimes_{\mathbb{R}} \mathbb{C}$ is multiplicity free as $G L_{2 n}(\mathbb{C})=G L_{n}(\mathbb{H}) \otimes_{\mathbb{R}} \mathbb{C}$ module (it is Proposition 2.1.7 of [A3] combined with Lemma 2.1.4 of [A3]; Proposition 2.1.7 of $\mathrm{A} 3$ is due to $[\mathrm{Ho}$, Proposition 2);

(c) $\Lambda_{I, \mathbb{R}}^{2 k, 0}(V) \otimes_{\mathbb{R}} \mathbb{C}$ and $\Omega^{k, k}(V) \otimes_{\mathbb{R}} \mathbb{C}$ are irreducible $G L_{2 n}(\mathbb{C})$-modules of the same highest weight.

Q.E.D.

From the construction of the product on $\Omega^{\bullet}(V)$ and the isomorphisms $\tau_{k}: \Lambda_{I, \mathbb{R}}^{2 k, 0}(V) \stackrel{\sim}{\rightarrow} \Omega^{k, k}(V)$ one easily has the following result.

\subsection{Proposition.}

$$
\oplus_{k=0}^{n} \tau_{k}: \oplus_{k=0}^{n} \Lambda_{I, \mathbb{R}}^{2 k, 0}(V) \stackrel{\sim}{\rightarrow} \Omega^{\bullet}(V)
$$

is an isomorphism of graded algebras where the algebra structure on

$$
\oplus_{k=0}^{n} \Lambda_{I, \mathbb{R}}^{2 k, 0}(V)
$$

is the usual wedge product.

In A3], Subsection 2.2, we have defined the cones $C^{k}(V)\left(\operatorname{resp} . K^{k}(V)\right)$ in $\Omega^{k, k}(V)$ of strongly (resp. weakly) positive elements. Definition [2.11 of cones in $\Lambda_{I, \mathbb{R}}^{2 k, 0}(V)$ in this article is obtained by applying the isomorphism $\tau_{k}$ to those cones in $\Omega^{k, k}(V)$. Hence Proposition 2.13 follows immediately from the corresponding properties of the cones in $\Omega^{\bullet}(V)$ (see Propositions 2.2.2-2.2.5 in [A3]). 
2.18 Proposition. The cone $C^{2}(V) \subset \Lambda_{I, \mathbb{R}}^{2,0}(V)$ is equal to the set $\{\eta \in$ $\left.\Lambda_{I, \mathbb{R}}^{2,0}(V) \mid \eta(A, A \circ J) \geq 0 \forall A \in V\right\}$.

Proof. Consider the cone $t\left(C^{2}(V)\right) \subset S_{\mathbb{H}}(V)$. This is the cone of strongly positive elements in $S_{\mathbb{H}}(V)$ considered in [A3. In the proof of Proposition 2.2.4 of A3 it was shown that this cone coincides with the cone of nonnegative definite hyperhermitian matrices. But $t(\eta)(A, A)=\eta(A, A \circ J)$. Proposition 2.18 is proved. Q.E.D.

\section{Differential operators on hypercomplex manifolds.}

Let $\left(X^{4 n}, I, J, K\right)$ be a hypercomplex manifold. Remind that we denote by $\Lambda_{I}^{p, q}$ the vector bundle of $(p, q)$-forms on the complex manifold $(X, I)$. By the abuse of notation we will denote by this symbol also the space of $C^{\infty}$-sections of this bundle.

Let

$$
\partial: \Lambda_{I}^{p, q}(X) \rightarrow \Lambda_{I}^{p+1, q}(X)
$$

be the usual $\partial$-differential of differential forms on the complex manifold $(X, I)$. Set

$$
\partial_{J}:=J^{-1} \circ \bar{\partial} \circ J
$$

3.1 Claim ([V2]; see also Claim 1.2]) $(1) J: \Lambda_{I}^{p, q}(X) \rightarrow \Lambda_{I}^{q, p}(X)$.

(2) $\partial_{J}: \Lambda_{I}^{p, q}(X) \rightarrow \Lambda_{I}^{p+1, q}(X)$.

(3) $\partial \partial_{J}=-\partial_{J} \partial$

3.2 Definition ([V2]). Let $k=0, \ldots, n$. A form $\omega \in \Lambda_{I}^{2 k, 0}(X)$ is called real if if it is real pointwise:

$$
\overline{J \circ \omega}=\omega .
$$

3.3 Lemma. Let $f: X \rightarrow \mathbb{R}$ be a real valued smooth function. Then $\partial \partial_{J} f \in$ $\Lambda_{I}^{2,0}(X)$ is real.

Proof.

$$
\begin{array}{r}
\overline{J \circ\left(\partial \partial_{J} f\right)}=\overline{J \circ \partial \circ J^{-1} \circ \bar{\partial} f}= \\
J \circ \bar{\partial} \circ J^{-1} \circ \bar{\partial} f=J \circ \bar{\partial} \circ J^{-1} \circ \partial \bar{f}=J \circ \bar{\partial} \circ J^{-1} \circ \partial f= \\
-\partial_{J} \partial f=\partial \partial_{J} f
\end{array}
$$


where the last equality is by Claim [3.1(3). Q.E.D.

Notation: (1) We will denote the subspace of real $C^{\infty}$-smooth $(2 k, 0)$ forms on $(X, I)$ by $\Lambda_{I, \mathbb{R}}^{2 k, 0}(X)$.

(2) We will denote by $S_{\mathbb{H}}(X)$ the vector bundle over $X$ with fiber over a point $x \in X$ equal to the space of hyperhermitian quadratic forms on the tangent space $T_{x} X$.

On the flat space $\mathbb{H}^{n}$ one can introduce so called Dirac operators. Let us describe them. We will write a quaternion $q$ in the usual form

$$
q=t+x \cdot i+y \cdot j+z \cdot k
$$

where $t, x, y, z$ are real numbers, and $i, j, k$ satisfy the usual relations

$$
i^{2}=j^{2}=k^{2}=-1, i j=-j i=k, j k=-k j=i, k i=-i k=j .
$$

The Dirac operator $\frac{\partial}{\partial \bar{q}}$ is defined as follows. For any $\mathbb{H}$-valued function $f$

$$
\frac{\partial}{\partial \bar{q}} f:=\frac{\partial f}{\partial t}+i \frac{\partial f}{\partial x}+j \frac{\partial f}{\partial y}+k \frac{\partial f}{\partial z}
$$

Let us also define the operator $\frac{\partial}{\partial q}$ :

$$
\frac{\partial}{\partial q} f:=\overline{\frac{\partial}{\partial \bar{q}} \bar{f}}=\frac{\partial f}{\partial t}-\frac{\partial f}{\partial x} i-\frac{\partial f}{\partial y} j-\frac{\partial f}{\partial z} k .
$$

It is easy to see that on $\mathbb{H}^{n}$

$$
\left[\frac{\partial}{\partial q_{i}}, \frac{\partial}{\partial \bar{q}_{j}}\right]=0 .
$$

It is easy to see that if $f: \mathbb{H}^{n} \rightarrow \mathbb{R}$ is a $C^{2}$-smooth function then the matrix $\left(\frac{\partial^{2} f}{\partial q_{i} \partial \bar{q}_{j}}\right)$ is hyperhermitian (see [A1] $)$.

\section{Comparison with the flat case.}

Consider $\mathbb{H}^{n}$ as a right $\mathbb{H}$-vector space equipped with the standard coordinate system.

4.1 Proposition. Let $f: \mathbb{H}^{n} \rightarrow \mathbb{R}$ be a real valued smooth function. Then

$$
t\left(\partial \partial_{J} f\right)=\frac{1}{4}\left(\frac{\partial^{2} f}{\partial q_{i} \partial \bar{q}_{j}}\right) .
$$


It is enough to prove the formula (4.1) pointwise. Since all the expressions involved are equivariant under translations it is enough to prove (4.1) at 0 . It is enough to show that the hyperhermitian quadratic forms $\left.\left(t\left(\partial \partial_{J} f\right)\right)\right|_{0}$ and $\left(\frac{\partial^{2} f}{\partial q_{i} \partial \bar{q}_{j}}\right)(0)$ coincide on each quaternionic line. Since all the operators in (4.1) are equivariant under the group $G L_{n}(\mathbb{H})$ it is enough to assume that this quaternionic line is equal to $\{(q, 0, \ldots, 0) \mid q \in \mathbb{H}\}$. Thus we may assume that $n=1$. Then we have

$$
\frac{\partial^{2} f}{\partial q \partial \bar{q}}=\frac{\partial^{2} f}{\partial t^{2}}+\frac{\partial^{2} f}{\partial x^{2}}+\frac{\partial^{2} f}{\partial y^{2}}+\frac{\partial^{2} f}{\partial z^{2}}
$$

when $q=t+x I+y J+z K$.

Let us compute the left hand side in (4.1). Let us identify $\mathbb{H}^{1} \simeq \mathbb{C}^{2}$ as follows: for $q=t+x I+y J+z K=(t+x I)+J(y-z I)$ define

$$
\begin{aligned}
& z_{1}:=t+x I \\
& z_{2}:=y-z I .
\end{aligned}
$$

Thus $q=z_{1}+J z_{2}$. We have

$$
\begin{gathered}
\frac{\partial}{\partial \bar{z}_{1}}=\frac{1}{2}\left(\frac{\partial}{\partial t}+\sqrt{-1} \frac{\partial}{\partial x}\right) \\
\frac{\partial}{\partial \bar{z}_{2}}=\frac{1}{2}\left(\frac{\partial}{\partial y}-\sqrt{-1} \frac{\partial}{\partial z}\right) .
\end{gathered}
$$

\subsection{Claim.}

$$
\begin{array}{r}
J^{-1} \circ d \bar{z}_{1}=-d z_{2}, J^{-1} \circ d z_{1}=-d \bar{z}_{2} \\
J^{-1} \circ d \bar{z}_{2}=d z_{1}, J^{-1} \circ d z_{2}=d \bar{z}_{1} .
\end{array}
$$

Proof is a straightforward computation. Q.E.D. 
Next we have

$$
\begin{aligned}
-\partial_{J} \partial f & =-\partial_{J}\left(\frac{\partial f}{\partial z_{1}} d z_{1}+\frac{\partial f}{\partial z_{2}} d z_{2}\right)=-J^{-1} \circ \bar{\partial}\left(\frac{\partial f}{\partial z_{1}}\left(J \circ d z_{1}\right)+\frac{\partial f}{\partial z_{2}}\left(J \circ d z_{2}\right)\right) \\
& =-J^{-1} \circ \bar{\partial}\left(\frac{\partial f}{\partial z_{1}} d \bar{z}_{2}-\frac{\partial f}{\partial z_{2}} d \bar{z}_{1}\right) \\
& =-J^{-1} \circ\left(\frac{\partial^{2} f}{\partial z_{1} \partial \bar{z}_{1}} d \bar{z}_{1} \wedge d \bar{z}_{2}-\frac{\partial^{2} f}{\partial z_{2} \partial \bar{z}_{2}} d \bar{z}_{2} \wedge d \bar{z}_{1}\right) \\
& =-\left(\frac{\partial^{2} f}{\partial z_{1} \partial \bar{z}_{1}}+\frac{\partial^{2} f}{\partial z_{2} \partial \bar{z}_{2}}\right) \cdot J^{-1} \circ\left(d \bar{z}_{1} \wedge d \bar{z}_{2}\right) \\
& =-\frac{1}{4}\left(\frac{\partial^{2} f}{\partial t^{2}}+\frac{\partial^{2} f}{\partial x^{2}}+\frac{\partial^{2} f}{\partial y^{2}}+\frac{\partial^{2} f}{\partial z^{2}}\right)\left(d z_{1} \wedge d z_{2}\right) \\
& =-\frac{1}{4} \frac{\partial^{2} f}{\partial q \partial \bar{q}}\left(d z_{1} \wedge d z_{2}\right) .
\end{aligned}
$$

Hence

$$
t\left(\partial \partial_{J} f\right)=\frac{1}{4} \frac{\partial^{2} f}{\partial q \partial \bar{q}} \cdot t\left(d z_{1} \wedge d z_{2}\right) .
$$

Hence in order to finish the proof of Proposition 4.1] it remains to prove the following claim.

\subsection{Claim.}

$$
t\left(d z_{1} \wedge d z_{2}\right)=d t^{2}+d x^{2}+d y^{2}+d z^{2} .
$$

Proof is a straightforward computation. Q.E.D.

4.4 Remark. The form $d z_{1} \wedge d z_{2} \wedge \cdots \wedge d z_{2 n-1} \wedge d z_{2 n}$ belongs to $\Lambda_{I, \mathbb{R}}^{2 n, 0}$ on $\mathbb{H}^{n}$.

Let us denote by $\underline{\mathbb{R}}$ the trivial real line bundle over $\mathbb{H}^{n}$. Let us consider the isomorphism of line bundles

$$
F: \Lambda_{I, \mathbb{R}}^{2 n, 0} \rightarrow \underline{\mathbb{R}}
$$

defined by

$$
F(\eta):=\frac{1}{n !} \frac{\eta}{d z_{1} \wedge d z_{2} \wedge \cdots \wedge d z_{2 n-1} \wedge d z_{2 n}} .
$$

Let us denote by

$$
\kappa: \operatorname{Sym}^{n}\left(\Lambda_{I, \mathbb{R}}^{2,0}\right) \rightarrow \Lambda_{I, \mathbb{R}}^{2 n, 0}
$$


the natural map of vector bundles given by

$$
\kappa\left(\eta_{1} \otimes \cdots \otimes \eta_{n}\right):=\eta_{1} \wedge \cdots \wedge \eta_{n} .
$$

Let us denote by $\operatorname{det}\left(X_{1}, \ldots, X_{n}\right)$ the mixed determinant of hyperhermitian matrices $X_{1}, \ldots, X_{n} \in S_{\mathbb{H}}\left(\mathbb{H}^{n}\right)$. By definition, it is a polarization of the Moore determinant. More precisely, the mixed determinant is a map $\operatorname{Sym}^{n}\left(S_{\mathbb{H}}\left(\mathbb{H}^{n}\right)\right) \rightarrow \mathbb{R}$ which is uniquely characterized by the following property: for any hyperhermitian matrix $Y \in S_{\mathbb{H}}\left(\mathbb{H}^{n}\right), \operatorname{det}(Y, \ldots, Y)$ is equal to the Moore determinant $\operatorname{det} Y$.

4.5 Proposition. The following diagram is commutative

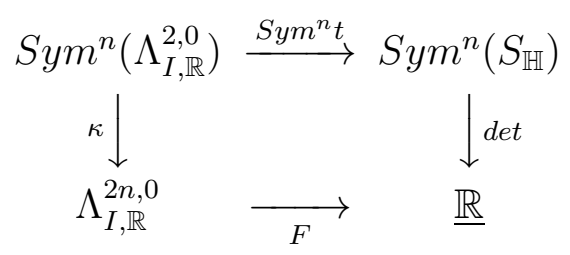

Proof. It is enough to check the commutativity of this diagram fiberwise. On each fiber we have the natural action of the group $S L_{n}(\mathbb{H})$ and all the maps commute with the action of this group (note that the action of this group on the spaces of the bottom line is trivial). By Lemma 2.1.4 of A3] and Proposition 2 of [Ho (which is cited in Proposition 2.1.7 of [A3]) the complexified representation of $G L_{n}(\mathbb{H})$ (and hence of $S L_{n}(\mathbb{H})$ ) in fibers of $\operatorname{Sym}^{n}\left(S_{\mathbb{H}}\right)$ is multiplicity free. Since the map $t$ commutes with the action of $G L_{n}(\mathbb{H})$, the complexified representation of $S L_{n}(\mathbb{H})$ in fibers of $\Lambda_{I, \mathbb{R}}^{2,0}$ is also multiplicity free. Thus the maps $\kappa$, det are characterized uniquely up to a constant by the property that they commute with the action of $S L_{n}(\mathbb{H})$. Hence the diagram (4.4) must be commutative up to a constant. To check the constant let us take $\left(d z_{1} \wedge d z_{2}\right) \otimes \cdots \otimes\left(d z_{2 n-1} \wedge d z_{2 n}\right)$. We have

$$
\begin{aligned}
& \operatorname{det}\left(\operatorname{sym}^{n} t\left(\left(d z_{1} \wedge d z_{2}\right) \otimes \cdots \otimes\left(d z_{2 n-1} \wedge d z_{2 n}\right)\right)\right)= \\
& \operatorname{det}\left(\left[\begin{array}{cccc}
1 & 0 & \ldots & 0 \\
0 & 0 & \ldots & 0 \\
\ldots & \ldots & \ldots \\
0 & 0 & \ldots & 0
\end{array}\right],\left[\begin{array}{cccc}
0 & 0 & \ldots & 0 \\
0 & 1 & \ldots & 0 \\
\ldots \ldots & \ldots . & \ldots \\
0 & 0 & \ldots & 0
\end{array}\right], \ldots,\left[\begin{array}{cccc}
0 & 0 & \ldots & 0 \\
0 & 0 & \ldots & 0 \\
\ldots \ldots & \ldots . & \ldots \\
0 & 0 & \ldots & 1
\end{array}\right]\right)= \\
& \left.\frac{1}{n !} \frac{\partial^{n}}{\partial \lambda_{1} \ldots \partial \lambda_{n}}\right|_{0} \operatorname{det}\left[\begin{array}{cccc}
\lambda_{1} & 0 & \ldots & 0 \\
0 & \lambda_{2} & \ldots & 0 \\
\ldots & \ldots \ldots & \ldots \\
0 & 0 & \ldots & \lambda_{n}
\end{array}\right]=\frac{1}{n !} \text {. }
\end{aligned}
$$


On the other hand

$$
\begin{array}{r}
\kappa\left(\left(d z_{1} \wedge d z_{2}\right) \otimes \cdots \otimes\left(d z_{2 n-1} \wedge d z_{2 n}\right)\right) \\
=\left(d z_{1} \wedge d z_{2}\right) \wedge \cdots \wedge\left(d z_{2 n-1} \wedge d z_{2 n}\right) .
\end{array}
$$

Proposition 4.5 is proved. Q.E.D.

From Propositions 4.1 and 4.5 we immediately get the following corollary.

\subsection{Corollary.}

$$
\left(\partial \partial_{J} f\right)^{n}=\frac{n !}{4^{n}} \operatorname{det}\left(\frac{\partial^{2} f}{\partial q_{i} \partial \bar{q}_{j}}\right) d z_{1} \wedge d z_{2} \wedge \cdots \wedge d z_{2 n-1} \wedge d z_{2 n} .
$$

\section{Vector bundles with a cone.}

5.1 Definition. Let $E \rightarrow X$ be a finite dimensional real vector bundle over a manifold $X$. We say that $E$ has a cone if at each fiber $E_{x}, x \in X$, we are given a convex cone $C_{x} \subset E_{x}$ with the following property: any point $x \in X$ has a neighborhood $U$, a trivialization $\phi:\left.E\right|_{U} \stackrel{\sim}{\rightarrow} X \times V$, and a convex cone $C \subset V$ such that for any $x \in U$ one has $\phi\left(C_{x}\right)=\{x\} \times C$.

Note that if a vector bundle $E$ has a cone then the dual bundle $E^{*}$ has a cone (which is dual to the cone of $E$ ).

Let us denote by $\left|\omega_{X}\right|$ the real line bundle of densities on $X$. It is canonically oriented. Hence if a bundle $E$ has a cone then naturally $E \otimes\left|\omega_{X}\right|$ has a cone.

5.2 Definition. (1) Let $E$ be a vector bundle with a cone. A continuous section $\gamma \in C(X, E)$ is called non-negative if at any point $x \in X \gamma(x) \in \bar{C}_{x}$ (where $\bar{C}_{x}$ denotes the closure of the cone $C_{x}$ ).

(2) A generalized section $\gamma \in C^{-\infty}(X, E)$ is called non-negative if for any non-negative section $\phi \in C_{0}^{\infty}\left(X, E^{*} \otimes\left|\omega_{X}\right|\right)$ one has $\gamma(\phi) \geq 0$.

It is easy to see that a continuous section $\gamma \in C(X, E)$ is non-negative if and only if it is non-negative as a generalized section.

5.3 Definition. Let $E$ be a vector bundle. An $E$-valued measure is a continuous linear functional $C_{0}\left(X, E^{*}\right) \rightarrow \mathbb{R}$. 
5.4 Proposition. Let $E$ be a vector bundle with a cone such that the dual bundle $E^{*}$ has a cone with non-empty interior at each point. Then any nonnegative generalized section of $E$ is an $E \otimes\left|\omega_{X}\right|^{*}$-valued measure.

Proof. This proposition is essentially well known. (When $E$ is the trivial line bundle this is proved in [GV], Ch. II.) Using partition of unity the proof reduces to the case of a trivial bundle $E$. Q.E.D.

Let us describe the now the main examples of bundles with a cone which will be used in this article. Let $\left(X^{4 n}, I, J, K\right)$ be a hypercomplex manifold. Let $0 \leq k \leq n$ be an integer. The bundle $\Lambda_{I, \mathbb{R}}^{2 k, 0}(X)$ is equipped with the cones $C^{k}(X)$ (resp. $K^{k}(X)$ ) of strongly (resp. weakly) positive elements as in Definition 2.11.

Let now $X=\mathbb{H}^{n}$ be the flat space. Consider the bundle $\Omega^{k, k}(X):=$ $X \times \Omega^{k, k}\left(\mathbb{H}^{n}\right)$ over $X, 0 \leq k \leq n$, where $\Omega^{k, k}\left(\mathbb{H}^{n}\right)$ is as in Proposition 2.14. This bundle is isomorphic to the bundle $\Lambda_{I, \mathbb{R}}^{2 k, 0}(X)$ via the isomorphism $\tau_{k}$ from Lemma 2.16. In A3. (see also the end of Section 2 of this article) we have described the cones of strongly and weakly positive elements in the bundle $\Omega^{k, k}(X)$. These cones correspond to the cones $C^{k}(X)$ and $K^{k}(X)$ in $\Lambda_{I, \mathbb{R}}^{2 k, 0}(V)(X)$ via the isomorphism $\tau_{k}$.

\section{Quaternionic plurisubharmonic functions on $\mathbb{H}^{n}$.}

In this section we will remind the notion and basic facts on quaternionic plurisubharmonic functions on $\mathbb{H}^{n}$ following [A1], A3].

Let $X$ be an open subset in $\mathbb{H}^{n}$.

6.1 Definition. A real valued function $u: X \rightarrow \mathbb{R}$ is called quaternionic plurisubharmonic if it is upper semi-continuous and its restriction to any right quaternionic line is subharmonic.

Recall that upper semi-continuity means that $u\left(x_{0}\right) \geq \limsup _{x \rightarrow x_{0}} u(x)$ for any $x_{0} \in X$. We will denote by $P(X)$ the class of plurisubharmonic functions in the open set $X$.

The class of all quaternionic plurisubharmonic functions in $X$ will be denoted by $P(X)$.

Sometimes we will abbreviate the term "quaternionic plurisubharmonic" by just "plurisubharmonic". 
6.2 Proposition ([A1]). Let $X \subset \mathbb{H}^{n}$ be an open subset. Let $f: X \rightarrow \mathbb{R}$ be a $C^{2}$-smooth function. Then

(1) the matrix $\left(\frac{\partial^{2} f}{\partial q_{i} \partial \bar{q}_{j}}\right)$ is hyperhermitian;

(2) the function $f$ is quaternionic plurisubharmonic if and only if this matrix $\left(\frac{\partial^{2} f}{\partial q_{i} \partial \bar{q}_{j}}\right)$ is non-negative definite.

Thus for any $C^{2}$-smooth function $f: X \rightarrow \mathbb{R}$ the matrix $\left(\frac{\partial^{2} f}{\partial q_{i} \partial \bar{q}_{j}}\right)$ is a continuous section of the bundle $\Omega^{1,1}(X)$. Let us denote for brevity

$$
D_{2} f:=\left(\frac{\partial^{2} f}{\partial q_{i} \partial \bar{q}_{j}}\right) .
$$

Thus by Proposition 6.2 $f$ is quaternionic plurisubharmonic function if and only if $D_{2} f$ take values in the cone of weakly (= strongly) positive elements of $\Omega^{1,1}(X)$.

6.3 Theorem ([A3]). Let $X \subset \mathbb{H}^{n}$ be an open subset. Let $0 \leq k \leq n$. For any functions $u_{1}, \ldots, u_{k} \in C(X) \cap P(X)$ one can define a $\bar{\Omega}^{k, k}(X) \otimes$ $\left|\omega_{X}\right|^{*}$-valued measure denoted by $D_{2} u_{1} \ldots D_{2} u_{k}$ with values in the cone of strongly positive elements which is uniquely characterized by the following two properties:

1) if $u_{1}, \ldots, u_{k} \in C^{2}(X)$ then the expression has the obvious meaning;

2) if sequences $\left\{u_{1}^{(N)}\right\}, \ldots,\left\{u_{k}^{(N)}\right\} \subset C(X) \cap P(X)$ are such that $u_{i}^{(N)} \rightarrow$ $u_{i} \in C(X) \cap P(X)$ uniformly on compact subsets of $X$ for any $i=1, \ldots, k$ then

$$
D_{2} u_{1}^{(N)} \cdot \ldots D_{2} u_{k}^{(N)} \stackrel{\text { weakly }}{\rightarrow} D_{2} u_{1} \cdot \ldots D_{2} u_{k}
$$

where the convergence is understood is the sense of the weak convergence of measures, i.e. in the space $\left(C_{c}\left(X,\left(\Omega^{k, k}(X)\right)^{*} \otimes\left|\omega_{X}\right|\right)\right)^{*}$ equipped with the weak topology.

Remarks. (a) It is easy to see that if $u_{N} \rightarrow u$ uniformly on compact subsets, and $u_{N} \in C(X) \cap P(X)$ then $u \in C(X) \cap P(X)$.

(b) Note that the real analogue of this result was proved by A.D. Aleksandrov [Al], and the complex analogue by Chern, Levine, and Nirenberg CLL.

(c) A special case of Theorem 6.3 with $k=n$ was proved earlier by one of the authors A1]. 


\section{Plurisubharmonic functions on hypercom- plex manifolds.}

Let $\left(X^{4 n}, I, J, K\right)$ be a hypercomplex manifold.

7.1 Definition. A continuous function

$$
h: X \rightarrow \mathbb{R}
$$

is called quaternionic plurisubharmonic (or just psh) if $\partial \partial_{J} h$ is a non-negative (generalized) section of $\Lambda_{I, \mathbb{R}}^{2,0}(X)$ (where the non-negativity is understood in the sense of Section 5 .

Let us denote by $P^{\prime}(X)$ the class of continuous psh functions on $X$. Let us denote by $P^{\prime \prime}(X)$ the class of functions from $P^{\prime}(X)$ with the following additional property: a function $h \in P^{\prime}(X)$ belongs to $P^{\prime \prime}(X)$ if and only if any $x \in X$ has a neighborhood $U \ni x$ and a sequence $\left\{h_{N}\right\} \subset P^{\prime}(U) \cap C^{2}(U)$ such that $h_{N} \stackrel{C^{0}(U)}{\rightarrow} h$. Thus $P^{\prime \prime}(X) \subset P^{\prime}(X)$.

7.2 Remark. If $X \subset \mathbb{H}^{n}$ is an open subset then it is easy to see using convolution with smooth non-negative functions that $P^{\prime \prime}(X)=P^{\prime}(X)=$ $P(X) \cap C(X)$ where $P(X)$ is the class of quaternionic plurisubharmonic functions as in Definition 6.1.

Conjecture. $P^{\prime \prime}(X)=P^{\prime}(X)$ on any hypercomplex manifold $X$.

7.3 Proposition. $P^{\prime \prime}(X)$ is closed under taking maximums.

To prove Proposition 7.3 we will need a lemma.

7.4 Lemma. Let $f, g \in P^{\prime}(X) \cap C^{2}(X), f, g>0$. Then for any $1 \leq p<\infty$

$$
\left(f^{p}+g^{p}\right)^{\frac{1}{p}} \in P^{\prime}(X) \cap C^{2}(X) .
$$

Let us deduce Proposition 7.3 from Lemma 7.4. Let $f, g \in P^{\prime \prime}(X)$. Since the statement is local, adding a large constant we may assume that $f, g>0$. Then there exist $\left\{f_{N}\right\},\left\{g_{N}\right\} \subset P^{\prime}(X) \cap C^{2}(X)$ such that $f_{N} \stackrel{C^{0}(X)}{\rightarrow} f, g_{N} \stackrel{C^{0}(X)}{\rightarrow}$ $g$. Moreover we may assume that $f_{N}, g_{N}>0$ for all $N$. Then $\max \left\{f_{N}, g_{N}\right\} \rightarrow$ $\max \{f, g\}$. It remains to show that $\max \left\{f_{N}, g_{N}\right\} \in P^{\prime \prime}(X)$. One has

$$
\max \left\{f_{N}, g_{N}\right\}=\lim _{p \rightarrow \infty}\left(f^{p}+g^{p}\right)^{\frac{1}{p}}
$$


Hence Lemma 7.4 implies Proposition [7.3.

Proof of Lemma 7.4. Step 1. Let us reduce the statement to the flat case $X=\mathbb{H}^{n}$. The plurisubharmonicity of $\left(f^{p}+g^{p}\right)^{\frac{1}{p}}$ for $f, g \in C^{2}(X)$ it is enough to check pointwise. Let us fix $p \in X$.

The following lemma is well known (see e.g. $[\mathrm{Sa}], \mathrm{Br}$ ) though we will outline a proof for convenience of the reader.

7.5 Lemma. Let $x_{0} \in X$. There exists a neighborhood $U \ni x_{0}$ and a diffeomorphism of $U$ onto an open subset $V \subset \mathbb{H}^{n}$ such that $x_{0}$ goes to 0 and

$$
\begin{array}{r}
I(x)=I_{0}+O\left(|x|^{2}\right) \\
J(x)=J_{0}+O\left(|x|^{2}\right) \\
K(x)=K_{0}+O\left(|x|^{2}\right)
\end{array}
$$

where $I_{0}, J_{0}, K_{0}$ are the standard quaternionic structures on $\mathbb{H}^{n}$.

Proof. The key point in the proof is the following result due to Obata Ob]: on the tangent bundle of hypercomplex manifold $(X, I, J, K)$ there exists unique torsion free connection $\nabla$ preserving the complex structure $I, J, K$ :

$$
\nabla I=\nabla J=\nabla K=0 .
$$

This connection is called the Obata connection.

Let us consider the geodesic coordinates with respect to the Obata connection $\nabla$ in a neighborhood of a point $x_{0} \in X$. Let us denote by $I_{0}, J_{0}, K_{0}$ the flat complex structure in this neighborhood. Thus

$$
I\left(x_{0}\right)=I_{0}\left(x_{0}\right), J\left(x_{0}\right)=J_{0}\left(x_{0}\right), K\left(x_{0}\right)=K_{0}\left(x_{0}\right) .
$$

Since the Obata connection is torsion free, the Cristoffel symbols of $\nabla$ vanish at $x_{0}$. Hence

$$
\left(\nabla I_{0}\right)\left(x_{0}\right)=\left(\nabla J_{0}\right)\left(x_{0}\right)=\left(\nabla K_{0}\right)\left(x_{0}\right)=0 .
$$

Then (7.1), (7.2) imply the proposition. Q.E.D.

It is enough to know $f, g$ in the 2-jet neighborhood of $x_{0}$, and the complex structures in 1-jet neighborhood of $x_{0}$. Hence by Lemma 7.5 we reduce Lemma 7.4 to the flat case.

Step 2. Assume that $X=\mathbb{H}^{n}$. Let us fix an arbitrary right $\mathbb{H}$-line $L$. We have to check that $\left.\left(f^{p}+g^{p}\right)^{\frac{1}{p}}\right|_{L}$ is subharmonic. Hence it remains to prove the following lemma. 
7.6 Lemma. Let $f, g>0$ be continuous subharmonic functions on a Euclidean space $L$. Then for any $1 \leq p<\infty$ the function $\left(f^{p}+g^{p}\right)^{\frac{1}{p}}$ is subharmonic.

To prove Lemma [7.6 (which is well known) let us fix a sphere $S$ with a center $x_{0} \in L$. Let $F(x):=(f(x), g(x)) \in \mathbb{R}^{2}$. We have

$$
\begin{gathered}
\left(f^{p}\left(x_{0}\right)+g^{p}\left(x_{0}\right)\right)^{\frac{1}{p}} \leq\left(\left(\int_{S} f(x)\right)^{p}+\left(\int_{S} g(x)\right)^{p}\right)^{\frac{1}{p}}= \\
\left\|\int_{S} F(x)\right\|_{l^{p}} \leq \int_{S}\|F(x)\|_{l^{p}}=\int_{S}\left(f(x)^{p}+g(x)^{p}\right)^{\frac{1}{p}} .
\end{gathered}
$$

Hence $\left(f^{p}+g^{p}\right)^{\frac{1}{p}}$ is subharmonic. Lemma [7.6 is proved. Hence Lemma 7.4 is proved too. Q.E.D.

7.7 Lemma. Let $X$ be a hypercomplex manifold, $\operatorname{dim}_{\mathbb{R}} X=4 n$. For any $\xi \in C_{0}^{\infty}\left(X, \Omega^{k}\right), \eta \in C_{0}^{\infty}\left(X, \Omega^{4 n-k-1}\right)$ one has

$$
\int_{X} \xi \wedge \partial_{J} \eta=-\int_{X} \partial_{J} \xi \wedge \eta .
$$

Proof. First observe that for any top degree form $\rho \in \Omega^{4 n}(X)$ one has

$$
J \circ \rho=\rho .
$$

Next for any forms $\alpha, \beta$ one has

$$
J \circ(\alpha \wedge \beta)=(J \circ \alpha) \wedge(J \circ \beta) .
$$

Using this and the Stokes formula we get

$$
\begin{array}{r}
\int \xi \wedge \partial_{J} \eta=\int \xi \wedge\left(J^{-1} \circ \bar{\partial} \circ J\right) \eta= \\
\int J \xi \wedge \bar{\partial} \circ J \circ \eta=-\int \bar{\partial} \circ J \circ \xi \wedge J \eta= \\
-\int J^{-1} \circ \bar{\partial} \circ J \circ \xi \wedge \eta=-\int \partial_{J} \xi \wedge \eta .
\end{array}
$$

Q.E.D. 
7.8 Proposition. Let $\left\{h_{N}\right\} \subset C(X)$. Let $h_{N} \stackrel{C^{0}}{\rightarrow} h$. Then

(1) if for any $N h_{N} \in P^{\prime}(X)$ then $h \in P^{\prime}(X)$;

(2) if for any $N h_{N} \in P^{\prime \prime}(X)$ then $h \in P^{\prime \prime}(X)$.

Proof. Part (2) easily follows from part (1). Thus let us prove part (1). We have to check that $h$ is psh. Let $\phi \in C_{0}^{\infty}\left(X, S_{\mathbb{H}}^{*} \otimes\left|\omega_{X}\right|\right) \simeq C^{\infty}\left(X, \Lambda_{I, \mathbb{R}}^{2 n-2,2 n}\right)$ be a non-negative section. Then by Lemma [7.7 one gets

$$
\begin{array}{r}
\int \partial \partial_{J} h \wedge \phi=\int h \partial \partial_{J} \phi= \\
\lim _{N \rightarrow \infty} \int h_{N} \partial \partial_{J} \phi=\lim _{N \rightarrow \infty} \int \partial \partial_{J} h_{N} \wedge \phi \geq 0 .
\end{array}
$$

The following result is an analogue of the theorems of Aleksandrov [A] and Chern-Levine-Nirenberg CLL.

7.9 Theorem. Let $0<k \leq n$. For any $h_{1}, \ldots, h_{k} \in P^{\prime \prime}(X)$ one can define a non-negative generalized section of $\Lambda_{I, \mathbb{R}}^{2 k}(X)$ denoted by $\partial \partial_{J} h_{1} \wedge \cdots \wedge \partial \partial_{J} h_{k}$ which is uniquely characterized by the following two properties:

(1) if $h_{1}, \ldots, h_{k} \in C^{2}(X)$ then the definition is clear;

(2) if $\left\{h_{i}^{N}\right\} \subset C^{2}(X), h_{i}^{N} \stackrel{C^{0}}{\rightarrow} h_{i}$ as $N \rightarrow \infty, i=1, \ldots, k$ then $h_{i} \in P^{\prime \prime}(X)$ and

$$
\partial \partial_{J} h_{1}^{N} \wedge \cdots \wedge \partial \partial_{J} h_{k}^{N} \rightarrow \partial \partial_{J} h_{1} \wedge \cdots \wedge \partial \partial_{J} h_{k}
$$

in the weak topology on $\left.\Lambda_{I, \mathbb{R}}^{2 k}(X) \otimes\left|\omega_{X}\right|^{*}\right)$-valued measures.

7.10 Remark. Theorem 6.3 is a special case of Theorem [7.9,

To prove Theorem [7.9 we will need a lemma.

7.11 Lemma. Let $0 \leq k \leq n$. Fix a compact subset $K \subset X$ and its compact neighborhood $\tilde{K} \supset K$. Then there exists a constant $C$ such that for any $h_{1}, \ldots, h_{k} \in P^{\prime}(X) \cap C^{2}(X)$ one has

$$
\left\|\partial \partial_{J} h_{1} \wedge \cdots \wedge \partial \partial_{J} h_{k}\right\|_{L^{1}(K)} \leq C \prod_{i=1}^{k}\left\|h_{i}\right\|_{C(\tilde{K})} .
$$

Proof of Lemma 7.11 Let us prove the lemma by induction in $k$. For $k=0$ the lemma is trivial. Let us assume that the lemma is true for $k-1$ 
and let us prove it for $k$. Let us fix a compact neighborhood $\hat{K}$ of $K$ such that $\tilde{K}$ is a neighborhood of $\hat{K}$ :

$$
K \subset \hat{K} \subset \tilde{K}
$$

Let us fix $\gamma \in C_{0}^{\infty}\left(X, \Lambda_{I}^{2 n-2 k, 2 n}(X)\right)$ which takes values in the cone of weakly positive elements, and moreover the restriction of $\gamma$ to $K$ takes values in the interior of this cone, and $\left.\gamma\right|_{X \backslash \hat{K}} \equiv 0$. Then

$$
\begin{array}{r}
\left\|\partial \partial_{J} h_{1} \wedge \cdots \wedge \partial \partial_{J} h_{k}\right\|_{L^{1}(K)} \leq C_{1} \int_{X} \partial \partial_{J} h_{1} \wedge \cdots \wedge \partial \partial_{J} h_{k} \wedge \gamma= \\
C_{1} \int_{X} h_{k} \partial \partial_{J}\left(\partial \partial_{J} h_{1} \wedge \cdots \wedge \partial \partial_{J} h_{k-1} \wedge \gamma\right)= \\
C_{1} \int_{\hat{K}} h_{k} \partial \partial_{J} h_{1} \wedge \cdots \wedge \partial \partial_{J} h_{k-1} \wedge \partial \partial_{J} \gamma \leq \\
C_{2}|| h_{k} \|_{C(\hat{K})} \int_{\hat{K}}\left|\partial \partial_{J} h_{1} \wedge \cdots \wedge \partial \partial_{J} h_{k-1} \wedge \partial \partial_{J} \gamma\right| \leq \\
C_{3}\left\|h_{k}\right\|_{C(\hat{K})}|| \partial \partial_{J} h_{1} \wedge \cdots \wedge \partial \partial_{J} h_{k-1}||_{L^{1}(\hat{K})} \leq C \prod_{i=1}^{k}|| h_{i} \|_{C(\tilde{K})}
\end{array}
$$

where the last inequality follows from the assumption of induction. Lemma 7.11 is proved. Q.E.D.

Proof of Theorem [7.9. Let $h_{1}, \ldots, h_{k} \in P^{\prime \prime}(X)$. Let us choose

$$
\left\{h_{i}^{N}\right\}_{N=1}^{\infty} \subset P^{\prime}(X) \cap C^{2}(X), i=1, \ldots, k
$$

such that $h_{i}^{N} \stackrel{C^{0}}{\rightarrow} h_{i}$ for each $i=1, \ldots, k$. Let us show that $\prod_{i=1}^{k} \partial \partial_{J} h_{i}^{N}$ converges weakly to a strongly non-negative measure with values in $\Lambda_{I}^{2 k, 0}(X)$. Since the $\Lambda_{I}^{2 k, 0}(X) \otimes\left|\omega_{X}\right|^{*}$-valued measures $\prod_{i=1}^{k} \partial \partial_{J} h_{i}^{N}$ are non-negative and locally bounded (by Lemma 7.11) there exists a subsequence $\left\{N_{l}\right\}$ such that $\prod_{i=1}^{k} \partial \partial_{J} h_{i}^{N_{l}}$ converges weakly to a non-negative $\Lambda_{I}^{2 k, 0}(X) \otimes\left|\omega_{X}\right|^{*}$-valued measure $\mu$. Let us show that $\mu$ does not depend on a choice of a convergent subsequence. Let us show it by induction in $k$. For $k=0$ this is trivial. Let us assume that the statement is true for $k-1$ and let us prove it for $k$. Let $\nu$ be another weak limit of some subsequence of the sequence $\left\{\prod_{i=1}^{k} \partial \partial_{J} h_{i}^{N}\right\}$. It is enough to check that for any $\phi \in C_{0}^{\infty}\left(X, \Lambda^{2 n-2 k, 2 n}(X)\right)$

$$
\int_{X} \mu \wedge \phi=\int_{X} \nu \wedge \phi .
$$


We have

$$
\begin{array}{r}
\int \mu \wedge \phi=\lim _{l \rightarrow \infty} \int \prod_{i=1}^{k} \partial \partial_{J} h_{i}^{N_{l}} \wedge \phi= \\
\lim _{l \rightarrow \infty} \int h_{k}^{N_{l}} \cdot\left(\prod_{i=1}^{k-1} \partial \partial_{J} h_{i}^{N_{l}}\right) \wedge \partial \partial_{J} \phi .
\end{array}
$$

By the assumption of induction the sequence $\left\{g_{N}:=\prod_{i=1}^{k-1} \partial \partial_{J} h_{i}^{N_{l}}\right\}$ is weakly convergent. Let us denote its weak limit by $g$. The sequence $\left\{f_{N}:=h_{k}^{N}\right.$. $\left.\partial \partial_{J} \phi\right\}$ has uniformly bounded support and converges uniformly (i.e. in $C^{0}$ topology) to $f:=h_{k} \cdot \partial \partial_{J} \phi$. Thus the existence of the limit in (7.5) follows from the following known lemma.

7.12 Lemma. Let $M$ be a compact topological space. Let $E \rightarrow M$ be a vector bundle. Let $\left\{f_{N}\right\} \subset C(M, E)$ be a sequence of sections which converges to $f \in C(M, E)$ uniformly on $M$. Let $\left\{g_{N}\right\} \subset C(M, E)^{*}$ be a sequence in the dual space which is weakly convergent to $g \in C(M, E)^{*}$. Then

$$
g_{N}\left(f_{N}\right) \rightarrow g(f) .
$$

Let us postpone the proof of this lemma which is well known. This lemma implies that $\lim _{N \rightarrow \infty} \int h_{k}^{N} \cdot\left(\prod_{i=1}^{k-1} \partial \partial_{J} h_{i}^{N}\right) \wedge \partial \partial_{J} \phi$ does exist, and by the same argument it should be equal to $\int \nu \wedge \phi$. Hence the equality (7.3) follows. Hence there exists a weak limit of the sequence $\prod_{i=1}^{k} \partial \partial_{J} h_{i}^{N}$.

It remains to show that if $h_{1}, \ldots, h_{k} \in P^{\prime}(X) \cap C^{2}(X)$ then the limit is equal to $\prod_{i=1}^{k} \partial \partial_{J} h_{i}$. Let us show this by induction in $k$. For $k=0$ this is trivial. Let us make the induction step. Let us denote by $\mu$ the weak limit of $\prod_{i=1}^{k} \partial \partial_{J} h_{i}^{N}$. Let us fix $\phi \in C_{0}^{\infty}\left(X, \Lambda^{2 n-2 k, 2 n}(X)\right)$. We have

$$
\begin{array}{r}
\int \mu \wedge \phi=\lim _{N \rightarrow \infty} \int \prod_{i=1}^{k} \partial \partial_{J} h_{i}^{N} \wedge \phi= \\
\lim _{N \rightarrow \infty} \int h_{k}^{N} \cdot \prod_{i=1}^{k-1} \partial \partial_{J} h_{i}^{N} \wedge \partial \partial_{J} \phi .
\end{array}
$$

By Lemma 7.12 the last limit is equal to

$$
\int h_{k} \cdot \prod_{i=1}^{k-1} \partial \partial_{J} h_{i} \wedge \partial \partial_{J} \phi .
$$


Let us show that (7.6) is equal to $\prod_{i=1}^{k} \partial \partial_{J} h_{i} \wedge \phi$. Using approximation and Lemma 17.12, we may assume that $h_{1}, \ldots, h_{k} \in P^{\prime}(X) \cap C^{2}(X)$. This case follows from Lemma [7.7. Theorem [7.9] is proved. Q.E.D.

Proof of Lemma 7.12. By the Banach-Steinhauss theorem the sequence $\left\{g_{N}\right\}$ in bounded in the $C(M, E)^{*}$-norm. Then we have

$$
\begin{array}{r}
\left|g_{N}\left(f_{N}\right)-g(f)\right| \leq\left|g_{N}\left(f_{N}-f\right)\right|+\left|\left(g_{N}-g\right)(f)\right| \leq \\
C|| f_{N}-f\left|\|_{C(M, E)}+\right|\left(g_{N}-g\right)(f) \mid \rightarrow 0 .
\end{array}
$$

Q.E.D.

\section{Quaternionic plurisubharmonic functions and HKT-geometry.}

In this section we present a geometric interpretation of quaternionic strictly plurisubharmonic functions on hypercomplex manifolds as local potentials of HKT-metrics (see Definition 1.12). This result is analogous to the classical well known fact that the complex strictly plurisubharmonic functions on complex manifolds are precisely local potentials of Kähler metrics.

Let $\left(X^{4 n}, I, J, K\right)$ be a hypercomplex manifold. Remind that we have the natural identification

$$
t: \Lambda_{I, \mathbb{R}}^{2,0}(X) \stackrel{\sim}{\rightarrow} S_{\mathbb{H}}(X)
$$

defined by (2.1) in Section 3, The following proposition which we call a local $\partial \partial_{J}$-lemma, is a rather straightforward application of the main result of [BS] (see also [B] ).

8.1 Proposition. Let $\Omega \in C^{\infty}\left(X, \Lambda_{I, \mathbb{R}}^{2,0}\right)$. Then locally on $X$ the form $\Omega$ can be presented in a form

$$
\Omega=\partial \partial_{J} f
$$

with $f$ being a $C^{\infty}$-smooth real valued function if and only if $\partial \Omega=0$.

Proof. The only if part is clear:

$$
\left.\partial\left(\partial \partial_{J}\right) \Omega\right)=\partial^{2}\left(\partial_{J} \Omega\right)=0
$$

Let us prove the converse statement. We will use heavily the result of [BS] (which is based in turn on [MCSa], see also [B]). The article [BS] uses a 
convention that the complex structures $I, J, K$ act on the left on the tangent vectors to $X$. Though in this article we use the opposite convention, in the proof of this proposition we will change our convention in order to cite the results of [BS] without change of signs and normalizations.

For a complex structure $\mathcal{I}$ compatible with the quaternionic structure (namely $\mathcal{I}$ has a form $\mathcal{I}=a I+b J+c K, a, b, c \in \mathbb{R}, a^{2}+b^{2}+c^{2}=1$ ) let us define the action of $\mathcal{I}$ on $k$ forms

$$
(\mathcal{I} \circ \omega)\left(X_{1}, \ldots, X_{k}\right)=\omega\left(\mathcal{I}^{-1} X_{1}, \ldots, \mathcal{I}^{-1} X_{k}\right)
$$

Define differentials on $k$-forms

$$
d_{\mathcal{I}} \omega:=-\mathcal{I}^{-1} \circ d \circ \mathcal{I} \circ \omega .
$$

Note that the operators $d_{\mathcal{I}}$ coincide with those considered in Section 2 of $[\mathrm{BS}]$. It is known (and one can be easily check it by a straightforward computation) that $d, d_{I}, d_{J}, d_{K}$ anticommute. Also one has

$$
\partial=\frac{1}{2}\left(d+\sqrt{-1} d_{I}\right), \bar{\partial}=\frac{1}{2}\left(d-\sqrt{-1} d_{I}\right) .
$$

Next one obtains

$$
\begin{array}{r}
\partial \partial_{J}=\frac{1}{4}\left(d+\sqrt{-1} d_{I}\right) \circ J^{-1} \circ\left(d-\sqrt{-1} d_{I}\right) \circ J= \\
\frac{1}{4}\left(d+\sqrt{-1} d_{I}\right)\left(J^{-1} \circ d \circ J+\sqrt{-1} J^{-1} I^{-1} \circ d \circ I J\right)= \\
\frac{1}{4}\left(d+\sqrt{-1} d_{I}\right)\left(-d_{J}-\sqrt{-1} d_{K}\right)= \\
-\frac{1}{4}\left(\left(d d_{J}-d_{I} d_{K}\right)+\sqrt{-1}\left(d d_{K}+d_{I} d_{J}\right)\right)= \\
-\frac{1}{4}\left(\left(d d_{J}+d_{K} d_{I}\right)+\sqrt{-1}\left(d d_{K}+d_{I} d_{J}\right)\right) .
\end{array}
$$

Thus we get

$$
\partial \partial_{J}=-\frac{1}{4}\left(\left(d d_{J}+d_{K} d_{I}\right)+\sqrt{-1}\left(d d_{K}+d_{I} d_{J}\right)\right) .
$$

Let us denote by $g:=t(\Omega)$ the smooth section of $\Lambda_{I, \mathbb{R}}^{2,0}(X)$ corresponding to $\Omega$. For a complex structure $\mathcal{I}$ let us define a 2 -form

$$
F_{\mathcal{I}}(X, Y)=g(\mathcal{I} X, Y)
$$


Then

$$
\Omega=c \cdot\left(F_{J}+\sqrt{-1} F_{K}\right)
$$

where $c$ is a non-zero normalizing constant which we will not write down explicitly. (Note also that in the right hand side of (8.2) we have the sign "plus" instead of "minus" as previously since now we work with left hypercomplex structures in the opposite to our previous conventions).

By the main theorem of [BS] locally on $X$ there exists an infinitely smooth function $\mu$ such that

$$
F_{I}=\frac{1}{2}\left(d d_{I}+d_{J} d_{K}\right) \mu .
$$

By Remark at the beginning part of Section 2 of [BS] the last identity is equivalent to each of the following two identities:

$$
\begin{aligned}
& F_{J}=\frac{1}{2}\left(d d_{J}+d_{K} d_{I}\right) \mu \\
& F_{K}=\frac{1}{2}\left(d d_{K}+d_{I} d_{J}\right) \mu .
\end{aligned}
$$

Substituting the last two identities into (8.2) we obtain

$$
\Omega=\frac{c}{2}\left(\left(d d_{J}+d_{K} d_{I}\right)+\sqrt{-1}\left(d d_{K}+d_{I} d_{J}\right)\right) \mu .
$$

Using (8.1) we deduce

$$
\Omega=c^{\prime} \cdot \partial \partial_{J} \mu \text {. }
$$

Thus Proposition 8.1 is proved. Q.E.D.

8.2 Proposition. (1) Let $f$ be an infinitely smooth strictly plurisubharmonic function on a hypercomplex manifold $(X, I, J, K)$. Then $t\left(\partial \partial_{J} f\right)$ is an HKTmetric.

(2) Conversely assume that $g$ is an HKT-metric. Then any point $x \in$ $X$ has a neighborhood $U$ and an infinitely smooth strictly plurisubharmonic function $f$ on $U$ such that $g=t\left(\partial \partial_{J} f\right)$ in $U$.

Proof. This is an immediate consequence of Proposition 8.1 and the definition of plurisubharmonic function. Q.E.D.

Non-negativity of a $(2,0)$-form $\rho \in \Lambda_{I, \mathbb{R}}^{2,0}(X)$ was defined and explored at great length in [V1], under the name " $K$-positivity". There it was related 
to a notion of stability for coherent sheaves on hyperkähler manifolds, and used to establish stability in some important cases. Quaternionic analogues of some fundamental results on positive currents were proven; in particular, a quaternionic version of Sibony's lemma on extensions ( $\underline{\text { Sib }}$ ) and a version of Skoda-El Mir theorem.

8.3 Theorem. Let $(X, I, J, K)$ be a hyperkähler manifold, $Z \subset(X, K)$ a complex subvariety, $\operatorname{codim}_{\mathbb{C}}(Z, X) \leq 3$, and $\rho \in \Lambda_{I, \mathbb{R}}^{2,0}(X)$ a $\partial$-closed nonnegative form on $X \backslash Z$. Then $\rho$ is locally $L^{1}$-integrable on $X$, and the corresponding current on $X$ is $\partial$-closed.

Proof: This is Proposition 7.5 from V1]. Q.E.D.

\section{References}

[Al] Aleksandrov, A. D.; Dirichlet's problem for the equation Det $\left\|z_{i j}\right\|=$ $\varphi\left(z_{1}, \cdots, z_{n}, z, x_{1}, \cdots, x_{n}\right)$. I. (Russian) Vestnik Leningrad. Univ. Ser. Mat. Meh. Astr. 131958 no. 1, 5-24.

[A1] Alesker, Semyon; Non-commutative linear algebra and plurisubharmonic functions of quaternionic variables. Bull. Sci. Math., 127 (2003), no. 1, 135. also: math.CV/0104209

[A2] Alesker, Semyon; Quaternionic Monge-Ampre equations. J. Geom. Anal. 13 (2003), no. 2, 205-238. also: math.CV/0208005.

[A3] Alesker, Semyon; Valuations on convex sets, non-commutative determinants, and pluripotential theory. Adv. Math. 195 (2005), 561-595. also: math.MG/0401219

[As] Aslaksen, Helmer; Quaternionic determinants. Math. Intelligencer 18 (1996), no. $3,57-65$.

[B] Baston, R. J.; Quaternionic complexes. J. Geom. Phys. 8 (1992), no. 1-4, $29-52$.

[BS] Banos, Bertrand; Swann, Andrew; Potentials for hyper-Kähler metrics with torsion. Classical Quantum Gravity 21 (2004), no. 13, 3127-3135. 
[Bo] Boyer, Charles P.; A note on hyper-Hermitian four-manifolds. Proc. Amer. Math. Soc. 102 (1988), no. 1, 157-164.

[Br] Bryant, Robert; Classical, exceptional, and exotic holonomies: a status report, in Actes de la Table Ronde de Géométrie Différentielle (Luminy, 1992), Sémin. Congr., vol. 1 (1996), pp. 93-165, Soc. Math. France, Paris.

[CLL] Chern, Shiing-shen; Levine, Harold I.; Nirenberg, Louis; Intrinsic norms on a complex manifold. 1969 Global Analysis (Papers in Honor of K. Kodaira) pp. 119-139 Univ. Tokyo Press, Tokyo.

[GP] Grantcharov, Gueo; Poon, Yat Sun; Geometry of hyper-Kähler connections with torsion, Comm. Math. Phys. 213 (2000), no. 1, 19-37.

[GV] Gelfand, I. M.; Vilenkin, N. Ya.; Generalized functions. Vol. 4. Applications of harmonic analysis. Translated from the Russian by Amiel Feinstein. Academic Press [Harcourt Brace Jovanovich, Publishers], New York-London, 1964 [1977].

[GRW] Gelfand, Israel; Retakh, Vladimir; Wilson, Robert Lee; Quaternionic quasideterminants and determinants. Lie groups and symmetric spaces, 111123, Amer. Math. Soc. Transl. Ser. 2, 210, Amer. Math. Soc., Providence, RI, 2003 .

[Ha] Harvey, Reese; Holomorphic chains and their boundaries. Several complex variables (Proc. Sympos. Pure Math., Vol. XXX, Part 1, Williams Coll., Williamstown, Mass., 1975), pp. 309-382. Amer. Math. Soc., Providence, R. I., 1977.

[He] Henkin, Gennadii; Private communication.

[Ho] Howe, Roger; Remarks on classical invariant theory. Trans. Amer. Math. Soc. 313 (1989), no. 2, 539-570.

[HP] Howe, P. S.; Papadopoulos, G.; Twistor spaces for hyper-Kähler manifolds with torsion. Phys. Lett. B 379 (1996), no. 1-4, 80-86.

[L] Lelong, Pierre; Fonctions plurisousharmoniques et formes différentielles positives. (French) Gordon \& Breach, Paris-London-New York (Distributed by Dunod éditeur, Paris) 1968.

[MCSa] Mamone Capria, M.; Salamon, S. M.; Yang-Mills fields on quaternionic spaces. Nonlinearity 1 (1988), no. 4, 517-530. 
[M] Moore,E.H.; On the determinant of an hermitian matrix of quaternionic elements. Bull. Amer. Math. Soc. 28 (1922), 161-162

[Ob] Obata, Morio; Affine connections on manifolds with almost complex, quaternion or Hermitian structure. Jap. J. Math. 26, 1956 43-77.

[Sa] Salamon, Simon M.; Differential geometry of quaternionic manifolds. Annales Scientifiques de l'cole Normale Suprieure Sr. 4, 19 no. 1 (1986), p. 31-55.

[Sib] Sibony, Nessim; Quelques problemes de prolongement de courants en analyse complexe, Duke Math. J. 52, 157-197 (1985).

[V1] Verbitsky, Misha; Hyperholomorpic connections on coherent sheaves and stability, 40 pages, math.AG/0107182

[V2] Verbitsky, Misha; HyperKähler manifolds with torsion, supersymmetry and Hodge theory. Asian J. Math. 6 (2002), no. 4, 679-712.

SEMyon Alesker

Department of Mathematics, Tel Aviv University, Ramat Aviv 69978 TEL AVIV, ISRAEL

semyon@post.tau.ac.il

Misha VERBITsKy

University of Glasgow, Department of Mathematics, 15 University Gardens, Glasgow G12 8QW, Scotland.

Institute of Theoretical And Experimental Physics

B. Cheremushininskaya, 25, Moscow, 117259, Russia

verbit@maths.gla.ac.uk, verbit@mccme.ru 